\title{
Avaliação do desempenho de escolares em testes de aptidão física
}

\author{
Juliano Maestri Alexandre ${ }^{1}$, Monalisa da Silva Reis², Renata Capistrano ${ }^{3}$, \\ Ana Paula Pietro Nobre Montoro ${ }^{4}$, Raísa Carvalho da Silva ${ }^{5}$, Thais Silva Beltrame ${ }^{6}$
}

\begin{abstract}
RESUMO
0 estudo teve como objetivo avaliar o desempenho de escolares em testes de aptidão física. A amostra foi composta por 88 escolares, dos quais 43 eram do sexo feminino e 45 do masculino. 0 instrumento utilizado foi à bateria de teste e medidas PROESP-BR contendo 5 itens: Teste de velocidade (corrida de $20 \mathrm{~m}$ ); Teste de agilidade (quadrado); Teste de força explosiva de membros inferiores (salto horizontal); Teste de força explosiva de membros superiores (arremesso de medicine ball) e resistência cardiorrespiratória (corrida de 6 minutos). Percebe-se que a maioria dos escolares apresentou níveis de aptidão física abaixo do esperado, principalmente nos componentes de força explosiva de membros inferiores, agilidade, velocidade e aptidão cardiorrespiratória. Sugere-se uma melhor caracterização dessa população, para que políticas públicas de incentivo a atividade física visando melhorar os índices de aptidão física de escolares possam ser criadas.
\end{abstract}

Descritores: Aptidão Física; Criança; Atividade Física.

\section{School performance assessment in physical fitness tests}

\begin{abstract}
The study aimed to evaluate the performance of students in physical fitness tests. The sample consisted of 88 students, of which 43 were female and 45 were male. The instrument used was to test battery and PROESP-BR measures containing 5 items: Speed test (20m running); Agility test (square); Explosive power test of the lower limbs (standing long jump); Explosive power test of the upper limbs (throwing medicine ball) and cardiorespiratory endurance (6-minute ride). It is noticed that most school showed levels of fitness lower than expected, especially in explosive force components of the lower limbs, agility, speed and cardiorespiratory fitness. It is suggested a better characterization of this population so that public policies to encourage physical activity to improve the physical fitness levels of school can be created.
\end{abstract}

Descriptors: Physical Fitness; Child; Physical Activity.

\footnotetext{
${ }^{1}$ Mestrando em Ciências do Movimento Humano pela Universidade do Estado de Santa Catarina (UDESC), Florianópolis, SC, Brasil.

2,4,5 Mestranda em Ciências do Movimento Humano pela Universidade do Estado de Santa Catarina (UDESC), Florianópolis, SC, Brasil.

3,6 Doutora em Ciências do Movimento Humano pela Universidade de Santa Maria (UFSM), Santa Maria, RS, Brasil.
} 


\section{Introdução}

A aptidão física é descrita como um componente biológico no qual possui a capacidade de executar atividades físicas com energia e vigor sem excesso de fadiga ${ }^{1,2}$. Diversos estudos apontam os benefícios de sua estimulação, ${ }^{3,45,6}$ porém nas últimas décadas, pode-se perceber uma grande alteração nos hábitos de estilo de vida da população. Com o crescente desenvolvimento das cidades, ocorreu uma diminuição na prática de atividades físicas, promovendo um declínio nos níveis satisfatórios de aptidão física, principalmente entre crianças e jovens ${ }^{7}$. Torna-se preocupante, visto que a literatura recomenda que o aprimoramento da aptidão física relacionada à saúde, tende a levar crianças e adolescentes a adotarem uma vida ativa ${ }^{8}$.

Por isso essa população na idade escolar têm se tornado objeto de estudo entre pesquisadores de todo mundo ${ }^{9-18}$. São pesquisas diversas nas áreas da ciência da motricidade humana, com forte contribuição ao estudo do crescimento, desenvolvimento e principalmente na aptidão física relacionada à saúde ${ }^{19}$.

Todavia, na literatura a aptidão física é apontada como dois componentes: relacionada à saúde e ao desempenho motor $^{20}$. Existem mais estudos relatando o primeiro componente, porém é imprescindível que a variável desempenho motor seja melhor abordada visto que contribui para o desempenho de tarefas específicas importantes para a vida ${ }^{21,22}$.

É necessária uma melhor contextualização acerca da aptidão física visto que escolares apresentam baixo nível de aptidão física relacionada ao desempenho motor ${ }^{22,23}$. Diante das considerações apresentadas, o presente estudo objetivou analisar a aptidão física relacionada ao desempenho motor de escolares de sete a dez anos de uma escola pública de Florianópolis.

\section{Metodologia}

Este estudo fez parte de um projeto intitulado "Desempenho motor, desenvolvimento físico, hábitos de vida em crianças de 7 a 10 anos." aprovado em 16 de junho de 2014 pelo Comitê de Ética e Pesquisa em Seres Humanos da Universidade do Estado de Santa Catarina (CAAE processo n 16449913.8.0000.0118).

Trata-se de um estudo descritivo-exploratório, no qual tem o objetivo de analisar determinados fenômenos, definir seus pressupostos, identificar suas estruturas ou esclarecer possíveis relações com outras variáveis ${ }^{24}$.

A amostra foi constituída por 88 escolares sendo 43 do sexo feminino e 45 do sexo masculino, entre sete e dez anos, aleatoriamente selecionados numa escola pública de ensino na cidade de Florianópolis, Santa Catarina.

Esses testes compreendem a avaliação da aptidão física relacionada ao desempenho motor (força explosiva dos membros superiores e inferiores, velocidade e agilidade e resistência geral).

$\mathrm{O}$ instrumento utilizado para avaliar o desempenho dos escolares foi a bateria de testes e medidas Proesp-br ${ }^{25}$, definise como um instrumentos de observação permanente dos indicadores de crescimento e desenvolvimento somatomotor e estado nutricional de crianças e adolescentes entre 6 a 17 anos.

A bateria de testes e medidas é constituida pelos testes de aptidão física relacionada a saúde e aptidão física relacionada ao desempenho, os testes relacionados à saúde estão associados à prevenção e a redução dos riscos de doenças, incluem testes de aptidão cardiorrespiratória (teste de corrida/caminhada de 6 minutos); composição corporal (IMC); teste da função músculo-esquelético (flexibilidade sentar e alcançar) e força/ resistência muscular (sit up). Já os testes de aptidão física relacioanda ao desempenho incluem potência de membros superiores (arremesso de medicineball); potência muscular de membros inferiores (salto horizontal); teste de agilidade (quadrado); teste de velocidade (corrida de 20 metros) e aptidão a cardiorrespiratória (corrida/caminhada de 6 minutos).

$\mathrm{O}$ estudo obedeceu à metodologia do instrumento (PROESP-BR), tendo como base valores numéricos que definem o nível de desempenho motor e estado nutricional dos adolescentes conforme a idade. A análise dos dados deu-se por meio da estatística descritiva, sendo classificados em uma escala de graus de aptidão variando de fraco a excelente.

\section{Resultados}

Participaram do estudo 88 escolares. Sendo 43 (48,86\%) do sexo feminino e $45(51,14 \%)$ do sexo masculino com idade de sete a dez anos conforme tabela 1. 
De acordo com as normas do proesp-br (2012) a avaliação da aptidão física relacionada ao desempenho está associada à prevenção e a redução dos riscos de doenças e a disposições para as atividades do dia-a-dia. Foi verificada a força explosiva de membros superiores e inferiores, agilidade, velocidade e aptidão cardiorrespiratória, pois são fundamentais para evitar 0 desenvolvimento de doenças, caracterizando por apresentar forte influência na prática da atividade física regular.

No teste de força explosiva de membros superiores foi possível observar que $19,32 \%$ dos escolares apresentaram nível fraco e razoável na mesma proporção, seguindo de 32,95\% nível bom, 26,41\% muito bom e 2,27\% excelente conforme tabela 1 .

Tabela 1 - Resultado do teste potência muscular de membros superiores

\begin{tabular}{c|c|c|c|c}
\hline $\begin{array}{c}\text { Força explosiva } \\
\text { de Membros } \\
\text { Superiores }\end{array}$ & $\begin{array}{c}\text { Meninas } \\
\mathbf{n}(\%)\end{array}$ & $\begin{array}{c}\text { Meninos } \\
\mathbf{n}(\%)\end{array}$ & $\begin{array}{c}\text { Total } \\
\mathbf{n}\end{array}$ & $\%$ \\
\hline Fraco & $8(18,60 \%)$ & $9(20,00 \%)$ & 17 & $19,32 \%$ \\
\hline Razoável & $8(18,60 \%)$ & $9(20,00 \%)$ & 17 & $19,32 \%$ \\
\hline Bom & $13(30,23 \%)$ & $16(35,56 \%)$ & 29 & $32,95 \%$ \\
\hline Muito bom & $13(30,23 \%)$ & $10(22,22 \%)$ & 23 & $26,14 \%$ \\
\hline Excelente & $1(2,33 \%)$ & $1(2,22 \%)$ & 2 & $2,27 \%$ \\
\hline
\end{tabular}

Em relação à força explosiva de membros inferiores, observou-se $63,64 \%$ dos adolescentes tiveram rendimento fraco de aptidão física, sendo $51,16 \%$ para o feminino e $75,56 \%$ para o masculino, $15,91 \%$ apresentaram nível razoável, $13,64 \%$ apresentaram nível bom, 4,55 \% apresentaram nível muito bom e 2,27\% apresentaram nível excelente de aptidão de acordo com tabela 2.

Tabela 2 - Resultado do teste potência muscular de membros inferiores

\begin{tabular}{c|c|c|c|c}
\hline $\begin{array}{c}\text { Força explosiva } \\
\text { de Membros } \\
\text { Inferiores }\end{array}$ & $\begin{array}{c}\text { Meninas } \\
\mathbf{n}(\%)\end{array}$ & $\begin{array}{c}\text { Meninos } \\
\mathbf{n}(\%)\end{array}$ & $\begin{array}{c}\text { Total } \\
\mathbf{n}\end{array}$ & $\%$ \\
\hline Fraco & $22(51,16 \%)$ & $34(75,56 \%)$ & 56 & $63,64 \%$ \\
\hline Razoável & $9(20,93 \%)$ & $5(11,11 \%)$ & 14 & $15,91 \%$ \\
\hline Bom & $8(18,60 \%)$ & $4(8,84 \%)$ & 12 & $13,64 \%$ \\
\hline Muito bom & $2(4,65 \%)$ & $2(4,44 \%)$ & 4 & $4,55 \%$ \\
\hline Excelente & $2(4,65 \%)$ & $0(0,00 \%)$ & 2 & $2,27 \%$ \\
\hline
\end{tabular}

O teste de agilidade $76,16 \%$ dos adolescentes classificou-se no nível fraco, sendo $74,42 \%$ meninas e $77,78 \%$ meninos, no entanto $10,23 \%$ dos adolescentes apresentaram nível razoável, 5,68\% nível bom e muito bom na mesma proporção e 2,27\% nível excelente. No geral, ambos os gêneros apresentam níveis de agilidade considerada como fraco de acordo com a tabela 3.

Tabela 3 - Resultado do teste de agilidade

\begin{tabular}{c|c|c|c|c}
\hline Agilidade & $\begin{array}{c}\text { Meninas } \\
\mathbf{n}(\%)\end{array}$ & $\begin{array}{c}\text { Meninos } \\
\mathbf{n}(\%)\end{array}$ & $\begin{array}{c}\text { Total } \\
\mathbf{n}\end{array}$ & $\%$ \\
\hline Fraco & $32(74,42 \%)$ & $35(77,78 \%)$ & 67 & $76,16 \%$ \\
\hline Razoável & $5(11,63 \%)$ & $4(8,89 \%)$ & 9 & $10,23 \%$ \\
\hline Bom & $2(4,65 \%)$ & $3(6,67 \%)$ & 5 & $5,68 \%$ \\
\hline Muito bom & $4(9,30 \%)$ & $1(2,22 \%)$ & 5 & $5,68 \%$ \\
\hline Excelente & $0(0,00 \%)$ & $2(4,44 \%)$ & 2 & $2,27 \%$ \\
\hline
\end{tabular}


Ao avaliar o teste de velocidade 42,05\% dos adolescentes obtiveram nível fraco, 41,68\% meninas e 42,22\%meninos, no entanto $18,18 \%$ nível razoável e bom na mesma proporção e 21,59\% nível muito bom conforme tabela 4.

Tabela 4 - Resultado do teste velocidade

\begin{tabular}{c|c|c|c|c}
\hline Velocidade & $\begin{array}{c}\text { Meninas } \\
\mathbf{n}(\%)\end{array}$ & $\begin{array}{c}\text { Meninos } \\
\mathbf{n}(\%)\end{array}$ & $\begin{array}{c}\text { Total } \\
\mathbf{n}\end{array}$ & $\%$ \\
\hline Fraco & $18(41,68 \%)$ & $19(42,22 \%)$ & 37 & $42,05 \%$ \\
\hline Razoável & $12(27,91 \%)$ & $4(8,89 \%)$ & 16 & $18,18 \%$ \\
\hline Bom & $8(18,60 \%)$ & $8(17,78 \%)$ & 16 & $18,18 \%$ \\
\hline Muito bom & $5(11,63 \%)$ & $14(31,11 \%)$ & 19 & $21,59 \%$ \\
\hline Excelente & $0(0,00 \%)$ & $0(0,00 \%)$ & 0 & $0,00 \%$ \\
\hline
\end{tabular}

Conforme os resultados da aptidão cardiorrespiratória observaram-se, que foi o teste com maior índice de nível fraco, tanto para meninos quanto para meninas $85,23 \%$ dos adolescentes encontram-se no nível indesejado de aptidão física "Fraco" sendo 79,07\% das meninas e $91,11 \%$ dos meninos, 10,23\% nível razoável, 1,14\% nível bom e 3,41\% nível muito bom como mostra a tabela 5 .

Tabela 5 - Resultado do teste aptidão cardiorrespiratória

\begin{tabular}{c|c|c|c|c}
\hline $\begin{array}{c}\text { Aptidão } \\
\text { Cardiorrespiratória }\end{array}$ & $\begin{array}{c}\text { Meninas } \\
\mathbf{n}(\%)\end{array}$ & $\begin{array}{c}\text { Meninos } \\
\mathbf{n}(\%)\end{array}$ & $\begin{array}{c}\text { Total } \\
\mathbf{n}\end{array}$ & $\%$ \\
\hline Fraco & $34(79,07 \%)$ & $41(91,11 \%)$ & 75 & $85,23 \%$ \\
\hline Razoável & $7(16,28 \%)$ & $2(4,44 \%)$ & 9 & $10,23 \%$ \\
\hline Bom & $1(2,33 \%)$ & $0(0,00 \%)$ & 1 & $1,14 \%$ \\
\hline Muito bom & $1(2,33 \%)$ & $2(4,44 \%)$ & 3 & $3,41 \%$ \\
\hline Excelente & $0(0,00 \%)$ & $0(0,00 \%)$ & 0 & $0,00 \%$ \\
\hline
\end{tabular}

\section{Discussão}

Diante dos resultados apresentados percebe-se que a maioria dos escolares apresentou desempenho indesejado para a aptidão nos seguintes componentes: aptidão cardiorrespiratória, agilidade, velocidade e força explosiva de membros inferiores.

Tais resultados podem ser explicados pelo fato de que um menor envolvimento com a prática de atividade física entre crianças e adolescentes vem crescendo nos últimos anos, devido a uma tendência ao sedentarismo ${ }^{26}$. Algumas atividades influenciam nessa tendência como assistir TV, navegar na internet, jogar vídeo game, entre outras ${ }^{27}$.

Um baixo nível de atividade física pode significar uma menor condição cardiorrespiratória. Nos últimos 45 anos, vem ocorrendo um declínio de $0,36 \%$ ao ano na aptidão cardiorrespiratória de crianças e adolescentes de todo mundo ${ }^{28}$. No presente estudo no teste de aptidão cardiorrespiratória os valores médios para a classificação "ruim" foram altos em ambos os sexos $(79,07 \%$ meninas e $91,11 \%$ meninos) assim como valores de excelentes mostraram-se baixos, podendo ser explicado ${ }^{29}$, pelo fato de que a baixa aptidão cardiorrespiratória está relacionada com o sedentarismo e atividades passivas.

A baixa aptidão física está associada a elevados índices de colesterol e triglicerídeos, pressão arterial e risco maior a obesidade, o que pode ser influenciado pelo estado socioeconômico, bem como por outros fatores, tanto ambientais, disponibilidade de espaços para realizar atividades, quanto sociais, influência dos amigos e pessoas próximas no comportamento relacionado à prática de atividade física ${ }^{30}$.

Quanto à Agilidade e Velocidade, nas idades de 7 a 10 anos os gêneros não apresentaram diferenças significativas, resultado semelhante são encontrados com estudo realizado em crianças Sul-Africanas de 8 e 9 anos $^{31}$, porém difere de um estudo que encontrou resultados estatisticamente significantes de um melhor desempenho dos meninos ${ }^{32}$. Estas valências são essenciais nas tarefas do dia-a-dia, como andar desviando de pessoas ou obstáculos, locomover-se carregando objetos ou deslocar-se rapidamente ${ }^{36}$. 
Com relação à força explosiva de membros inferiores, foi encontrado um elevado índice de escolares classificados como "ruim", em estudo realizado com crianças e adolescentes entre 7 a 16 anos de São Paulo, observou-se que mais de 50\% de sua população nessa classificação não havendo grandes diferenças entre os sexos, porém na tarefa, força explosiva de membros superiores, os resultados distribuíram-se pelas classificações em ambos os gêneros ${ }^{33}$. A literatura relata que a diferença entre gêneros para essas variáveis começa aparecer por volta dos 12 a 14 anos com a chegada da puberdade ${ }^{33,34,20}$.

Ao analisarmos cada teste realizado, constatamos que a força explosiva de membros superiores obteve melhores resultados, com menor percentual de índices insatisfatórios, indo de encontro com os achados no estudo ${ }^{33}$. No entanto no estudo realizado em São Paulo, encontrou diferentes resultados aos do achado do presente estudo, níveis muito baixos no teste de força explosiva de membros superiores, no qual tanto meninos quanto meninas foram avaliados, em sua maioria, na classificação de pior desempenho ou seja resultados insatisfatórios ${ }^{35}$.

Essas diferenças de desempenho motor podem ser influenciadas por três fatores: individual (biológico), ambiental (experiências) e da tarefa (físico-mecânicos) ${ }^{37}$. No entanto para obter a melhora dos índices nos aspectos do desempenho motor em escolares faz-se necessário a intervenção do professor de educação física, que deve destinar parte da programação de sua aula para o desenvolvimento dessas valências ${ }^{38}$.

\section{Considerações Finais}

Conclui-se que os apresentaram índices inferiores aos pontos de corte, definido pelo protocolo. As causas relacionadas a esse baixo nível de aptidão física necessitam ser mais esclarecidas. Inferimos ser necessária uma melhor caracterização dessa população, para que políticas públicas de incentivo a atividade física visando melhorar os índices de aptidão física de escolares possam ser criadas.

\section{Referências Bibliográficas}

1. Guedes DP, Neto JTM, Germano JM, Lopes V, Silva AJRM. Aptidão física relacionada à saúde de escolares: programa fitnessgram; Health-related physical fitness of schoolchildren: the fitnessgram program. Rev. bras. med. esporte. 2012; 18(2): $72-6$. 2. Silva RJS, Silva AGJ, Cabral ACO. Crescimento em crianças e adolescentes: um estudo comparativo. Rev Bras Cineantropom Desempenho Hum. 2005; 7(2): 55-61.

3. Ronque VER, Cyrino ES, Dórea V, Junior HS, Galdi EHG, Arruda M. Diagnóstico da aptidão física em escolares de alto nível socioeconômico: avaliação referenciada por critérios de saúde. Rev Bras Med Esporte. 2007; 13(2): 71-6.

4. Gonzáles F, Fensterseifer PE. Dicionário crítico da Educação Física. ljuí: Unijuí. RBCE. 2010; 10-21.

5. O'Donovan G, Blazevich AJ, Boreham C, Cooper AR, Crank H, Ekelund U et al. The abc of physical activity for health: a consensus statement from the British Association of Sport and Exercise Sciences. J Sports Sci. 2010.

6. Haskell WL, Lee IM, Pate RR, Powell KE, Blair SN, Franklin BAetal. Physical activity and public health: updated recommendation for adults from the American College of Sports Medicine and the American Heart Association. Med Sci Sports Exerc. 2007.

7. Rodrigues LP, Bezerra P, Saraiva L. Influência do meio (urbano e rural) no padrão de aptidão física de rapazes de Viana do Castelo, Portugal. Revista portuguesa de ciências do desporto 2005; 5(1): 77-84.

8. Bergmann GG, Araújo MLB, Garlipp DC, Lorenzi TDC, Gaya A. Alteração anual no crescimento e na aptidão física relacionada à saúde de escolares. Rev Bras Cineantropom Desempenho Hum 2005; 7(2):55-61.

9. Powell KE, Roberts AM, Ross JG, Phillips MAC, Ujamaa DA, Mei Zhou MA. Low physical fitness among fifth- and seventh-grade students, Georgia, 2006. Am J Prev Med 2009.

10. Welk GJ, Jackson AW, Morrow Junior JR, Haskell WH, Meredith MD, Cooper KH. The association of health-related fitness with indicators of academic performance in Texas schools. Res Q Exerc Sport 2010.

11. Welk GJ, Meredith MD, Ihmels M, Seeger C. Distribution of health-related physical fitness in Texas youth: a demographic and geographic analysis. Res Q Exerc Sport 2010.

12. Huberty JL, Rosenhranz RR, Balluff MA, High R. Describing weight status and fitness in a community sample of children attending after-school programming. J Sports Med Phys Fitness 2010. 
13. Shriver LH, Harrist AW, Hubbs-Tait L, Topham G, Page M, Barrett A. Weight status, physical activity, and fitness among third-grade rural children. J Sch Health 2011.

14. Coledam DHC, Arruda GA, Oliveira AR. Efeitos de um programa de exercícios no desempenho de crianças nos testes de flexibilidade e impulsão vertical. Motriz. Revista de Educação Física. 2012; 18(3): 515-525.

15. Burgos MS, Reuter CP, Tornquist L, Reckziegel MB, Pohl HH, Burgos LT. Perfil de aptidão física relacionada à saúde de crianças e adolescentes de 7 a 17 anos. Health Sci. Inst. 2012; 30(2).

16. Pereira CH, Ferreira DS, Copetti GL, Guimarães LC, Barbacena MM, Liggeri N et al. Aptidão física em escolares de uma unidade de ensino da rede pública de Brasília-DF. Revista Brasileira de Atividade Física \& Saúde. 2012; 16 (3):223-227.

17. Tornquist D, Tornquist L,Reuter CP, , Reckziegel MB, Burgos LT, Burgos MS . Physical aptitude related to the health of initial graders: A study between groups assisted and non assisted by a Physical Education professional. Revista Brasileira de Atividade Física \& Saúde 2013; 18(3).

18. Araújo SS, Oliveira ACC. Aptidão física em escolares de Aracaju. Rev. bras. cineantropom. desempenho hum. 2008; 10(3):271-276. 19. Guedes DP. Implicações associadas ao acompanhamento do desempenho motor de crianças e adolescentes. Rev Bras Educ Fis Esporte. 2007; 21:37-60.

20. Nieman DC. Exercício e saúde: como se prevenir de doenças usando o exercício como seu medicamento. Tradução de Marcos Ikeda. São Paulo: Manole, 1999; 3-20.

21. Nahas MV. Atividade física, saúde e qualidade de vida: conceitos e sugestões para um estilo de vida ativo. 2a ed. Midiograf; 2001.

22. Jochims S, Zeni AE, Nunes HMB, Borfe L, Burgos MS. Aptidão física relacionada ao desempenho motor de escolares: estudo comparativo dos hemisférios Norte-Sul-Leste-Oeste, da zona rural de Santa Cruz do Sul-RS. Cinergis. 2014; 14(2). 23. Solo AB. Aptidão física relacionada ao desempenho motor em escolares de sete a 15 anos. Rev. bras. Educ. Fís. Esporte. 2010; 24(1):5-14.

24. Thomas JR, Nelson JK. Métodos de Pesquisa em Atividade Física. 3a ed. Artmed; 2002.

25. Gaya A. Manual do Projeto Esporte Brasil, 2012.

26. Guerra C, Reckziegel MB, Burgos MS. Perfil somatomotor e indicadores de saúde de adolescentes com índices alterados de glicemia. Rev. Digital Cinergi. 2008; 9(1): 29-36.

27. Nobre FSS, Krebs RJ, Valentini NC. Práticas de lazer, nível de atividade física e aptidão física de moças e rapazes Brasileiros. Revsalud pública. 2009; 11(5): 713-723.

28. Tomkinson GR, Olds TS. Pediatric Fitness. Secular Trends and Geographic Variability.Med Sport Sci Basel. 2007;50:67-90.

29. Andersen LB, Van Mechelen W. Are children of today less active than before and is their health indanger. What can we do? Scand J Med Sci Sports.2005; 15:268-70.

30. Dumith SC, Ramires WV, Souza MJA, Moraes DS, Petry FG, Oliveira ES et al. Apidão física relacionada ao desempenho motor em escolares de sete a 15 anos. Rev. bras. Educ. Fís. Esporte. 2010; 24(1): 5-14.

31. Armstrong MEG, Lambert EV, Lambert MI. Physical fitness of south African primary school children, 6 to 13 years of age: discovery vitality health of the nation study 1,2. Perceptual and motor skills. 2011; 113(3): 999-1016.

32. Sacchetti R, Ceciliani A, Garulli A, Masotti A, Poletti G, Beltrami P et al. Physical fitness of primary school children in relation to overweight prevalence and physical activity habits. Journal of sports sciences. 2012; 30(7): 633-640.

33. Luguetti CN, Ré AHN, Böhme MTS. Indicadores de aptidão física de escolares da região centro-oeste da cidade de São Paulo. RevBrasCineantropom Deาsempenho Hum. 2010;12:331-337.

34. Lorenzi T, Garlipp D, Bergmann G, et al. Aptidão física relacionada ao desempe $\urcorner n h o$ motor de crianças e adolescentes do Rio Grande do Sul. Dossiê PROJETO ESPORTE RS. Revista Perf. 2005; 22-30.

35. Borges AF, Borim JP, Marco A. Avaliação de indicadores antropométricos e neuromusculares de jovens escolares do ensino fundamental do interior paulista. Motriz. 2010;16(2):326-37.

36. Miyasike-Da-Silva V, Villar R, Zago AS, Polastri PF, Gobbi S. Nível de agilidade em indivíduos entre 42 e 73 anos: efeitos de um programa de atividades físicas generalizadas de intensidade moderada. RBCE. 2002;23(3):65-79.

37. Gallahue DL, Ozmun JC. Compreendendo o desenvolvimento motor: bebês, crianças, adolescentes e adultos. São Paulo: Phoerte; 2001.

38. Verardi CEL, Lobo APS, Amaral VE, Freitas VL, Hirota VB. Análise da aptidão física relacionada à saúde e ao desempenho motor em crianças e adolescentes da cidade de Carneirinho-MG. Rev. Mackenzie Educ Fís Esporte. 2007;6(3):127-134. 


\section{Juliano Maestri Alexandre}

Endereço para correspondência - Rua: Pascoal Simone, Bairro: Coqueiros, CEP: 88080-350, Florianópolis, SC, Brasil.

E-mail: julianom.alexandre@gmail.com

Lattes: http://lattes.cnpq.br/4233615344781061

Monalisa da Silva Reis - monalisareis-bassal@hotmail.com

Renata Capistrano - recapis@gmail.com

Ana Paula Pietro Nobre Montoro - anappnmontoro@hotmail.com

Raísa Carvalho da Silva - rahisac@gmail.com

Thais Silva Beltrame - tsbeltrame@gmail.com

Enviado em 21 de novembro de 2014.

Aceito em 16 de setembro de 2015. 
